\title{
A case of primary adenosquamous carcinoma of the liver
}

\author{
Gu Hyum Kang ${ }^{1}$, Byung Seok Lee ${ }^{1}$, and Dae Young Kang ${ }^{2}$
}

Departments of ${ }^{1}$ Internal Medicine and ${ }^{2}$ Pathology, Chungnam National University School of Medicine, Daejeon, Korea

\begin{abstract}
Adenosquamous carcinoma (ASC) is a rare tumor of the liver, which carries a poor prognosis. It has been reported mostly in the form of case studies. Recently, we experienced a case of primary ASC of the liver that has been preoperatively considered as cholangiocelluar carcinoma of the liver. Microscopically, the tumor was composed of adenocarcinoma, squamous cell carcinoma, as well as transitional area, including the transformation of adenocarcinoma to squamous cell carcinoma. The patient remained well with no evidence of post-resection complication or recurrence for 15 month. (Korean J Hepatobiliary Pancreat Surg 2013;17:38-41)
\end{abstract}

Key Words: Cholangiocelluar carcinoma; Adenosquamous carcinoma; Liver

\section{INTRODUCTION}

Adenosquamous carcinoma (ASC) is a tumor composed of both adenocarcinoma and squamous cell carcinoma components, especially adenocarcinoma containing significant amounts of unequivocal squamous carcinomatous elements, for example keratin and/or intercelluar bridges. Primary ASC is considered as a subtype of cholangiocelluar carcinoma (CCC). The incidence of ASC is rather common in the gall bladder, pancreas, stomach, thyroid, and large intestine, but hepatic ASC is very rare. Primary ASC of the liver has been reported mostly in a form of case studies in South Korea. ASC tends to present more aggressive clinicopathologic features and has a worse prognosis than CCC. ${ }^{1,2}$ From five hepatobiliary ASC for the past 10 years in our institute, one primary hepatic ASC was observed. We describe a case of primary hepatic ASC, preoperatively diagnosed as CCC, along with investigating the literature of primary hepatic ASC and attempting to explore it clinicopathologically.

\section{CASE}

A 73-year-old man complained of fever and chill, and was hospitalized for urinary tract infection at the local clinic. During hospitalization, hepatic mass was observed from ultrasonography (US) and was transferred to Chungnam National University Hospital for further investigation of the liver mass. He had a history of endoscopic retrograde cholangiopancreatography (ERCP) and endoscopic sphincterotomy with stone extraction, due to cholangitis accompanying bile duct stone 6 years ago. He had neither specific family history nor history of smoking, as well as drinking. He was currently working in the field of agriculture. On admission, he was chronic ill looking with icteric sclera. He had a temperature of $36.2^{\circ} \mathrm{C}$ and physical examination was unremarkable.

Initial laboratory evaluation were as follows: WBC $7,880 / \mathrm{mm}^{3}, \mathrm{Hb} 13.1 \mathrm{~g} / \mathrm{dl}$, platelet $258,000 / \mathrm{mm}^{3}$, PT (prothrombin time) $12.5 \mathrm{sec}$ (INR: 1.1), aPTT (partial thromboplastin time) $23.6 \mathrm{sec}$, total protein $7.5 \mathrm{mg} / \mathrm{dl}$, Albumin $3.5 \mathrm{mg} / \mathrm{dl}$, AST $13 \mathrm{IU} / \mathrm{L}$, ALT $8 \mathrm{IU} / \mathrm{L}$, ALP $151 \mathrm{IU} / \mathrm{L}$, GGT (gamma glutamyl transferase) $89 \mathrm{IU} / \mathrm{L}$, total bilirubin $1.4 \mathrm{mg} / \mathrm{dl}$. HBsAg and HBsAb were both negative and HCV Ab was negative. CA 19-9 (carbohydrate antigen 19-9) and CEA (carcinoembryonic antigen) were $1,600 \mathrm{U} / \mathrm{ml}$ and $7.2 \mathrm{ng} / \mathrm{ml}$. Abdominal computed tomography $(\mathrm{CT})$ from the local clinic showed low-density mass with irregular margin on the left lobe. Hepatic mass showed rim enhancement on early phase with slow central

Received: June 5, 2012; Revised: August 27, 2012; Accepted: October 15, 2012

Corresponding author: Byung Seok Lee

Department of Internal Medicine, Chungnam National University Hospital, 640, Daesa-dang, Jung-gu, Daejeon 301-721, Korea

Tel: +82-42-280-8049, Fax: +82-42-257-5753, E-mail: gie001@cnuh.co.kr 

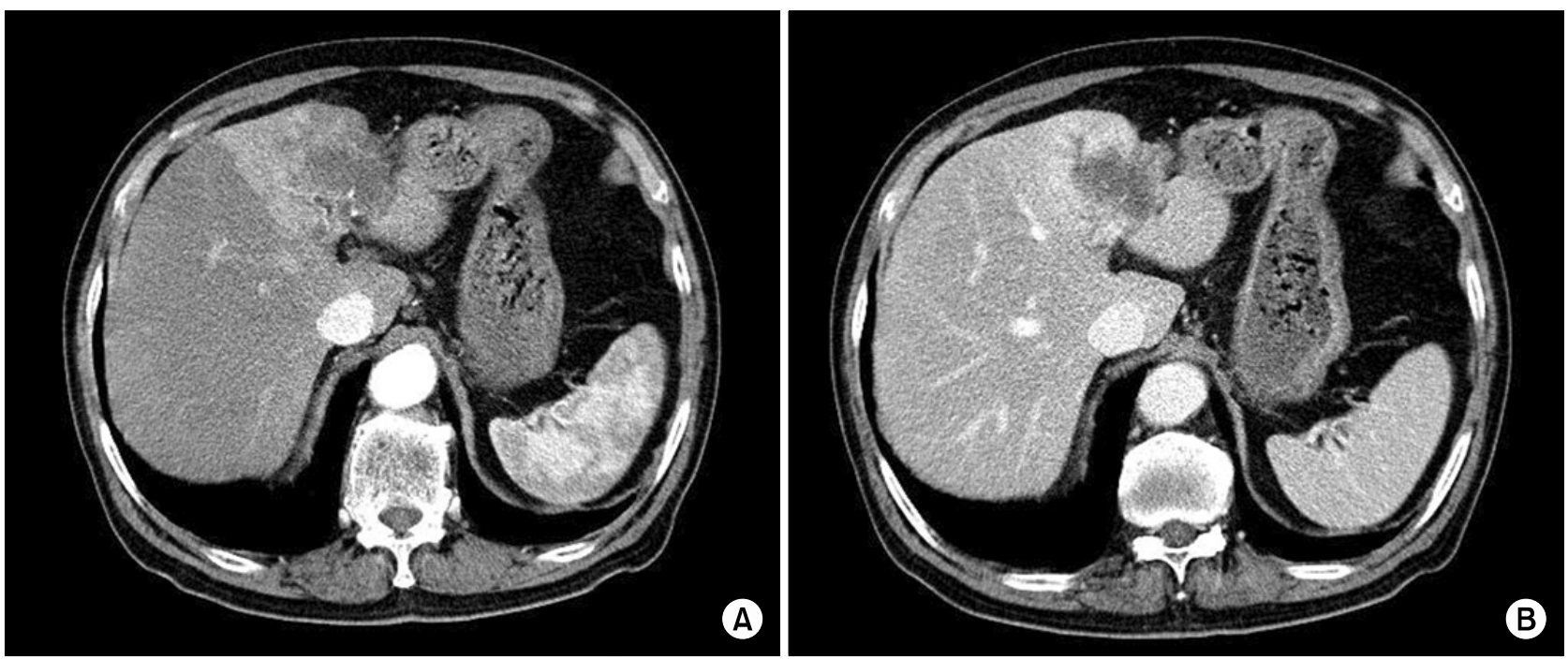

Fig. 1. Abdomen computed tomography shows a hypo-dense mass with irregular margin on the left hemiliver with early rim enhancement in the arterial phase (A) and slow central enhancement on delayed image (B).
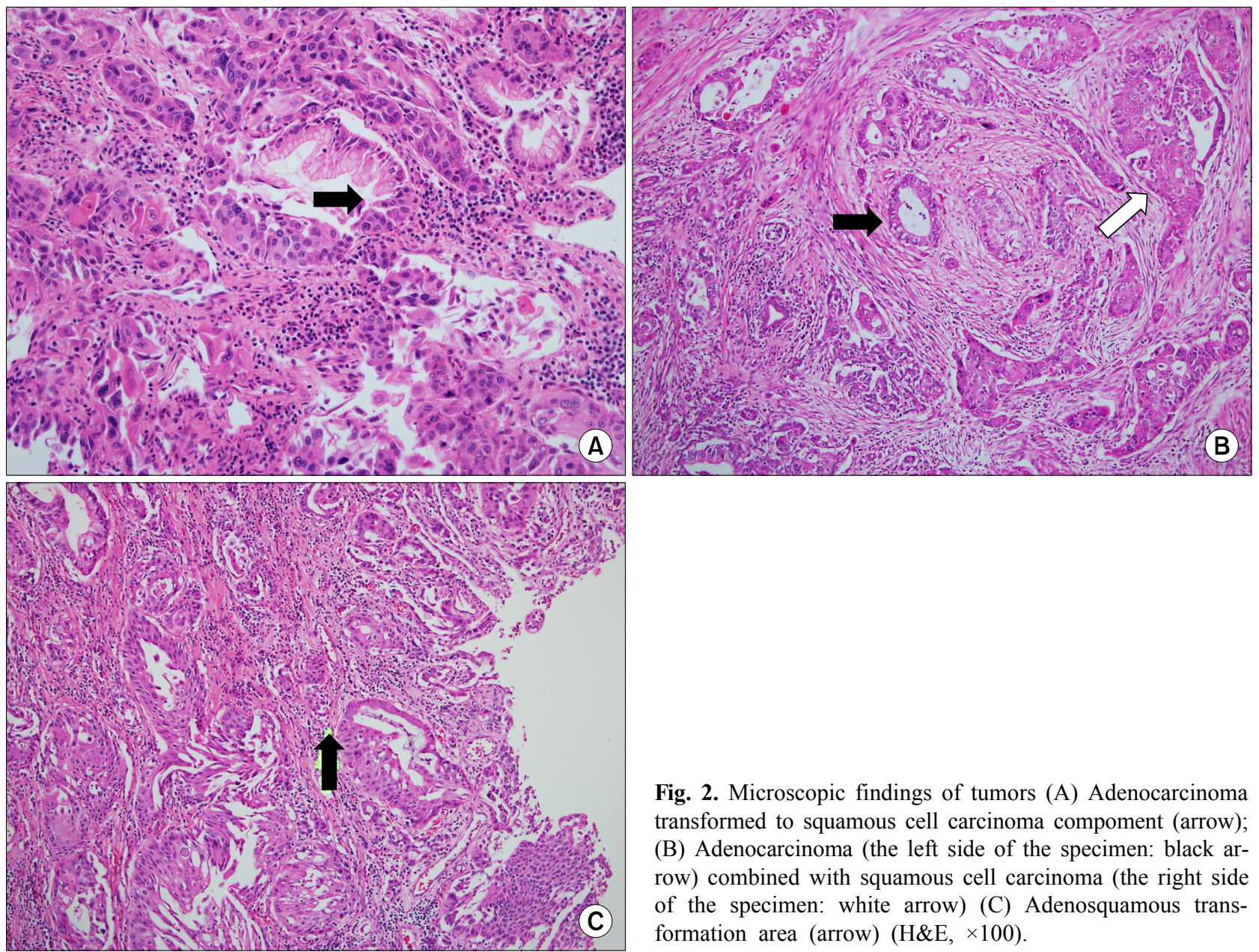

Fig. 2. Microscopic findings of tumors (A) Adenocarcinoma transformed to squamous cell carcinoma compoment (arrow); (B) Adenocarcinoma (the left side of the specimen: black arrow) combined with squamous cell carcinoma (the right side of the specimen: white arrow) (C) Adenosquamous transformation area (arrow) $(\mathrm{H} \& \mathrm{E}, \times 100)$. 
enhancement on delayed phases. Distal intrahepatic duct dilatation and cirrhotic change were observed (Fig. 1). Magnetic resonance imaging study showed a $5 \mathrm{~cm}$-sized lobulated mass with dilatation of peritumoral intrahepatic duct (IHD) in the left segment of the liver, which was consistent with hepatic CCC and no evidence of any abnormal lymphadenopathy in significant size.

Left hemihepatectomy and caudate lobectomy was conducted because the lesion seemed to be malignant. At operation, the tumor occupied II and III segment of the liver with periductal infiltration and intraductal growing to hilum. The specimen contained round yellow-gray cystic mass, measuring $5 \times 5 \mathrm{~cm}$. The tumor had free resection margin and lymph node labeled hepatoduodenal ligament and No. 8 had tumor metastasis. Postoperative TNM stage was pT1N1M0.

Microscopically, the tumor was composed of adenocarcinoma component and squamous cell carcinoma component with a few keratin pearls. Thus, a diagnosis of hepatic ASC could be made. This tumor was composed of adenocarcinoma, squamous cell carcinoma, as well as transitional area, including the transformation of adenocarcinoma to squamous cell carcinoma (Fig. 2). Postoperative adjuvant chemotherapy was performed, using 5-fluorouracil and leucovorin regimen. The patient remained well with no evidence of post operation complication or recurrence for 15 months since her operation.

\section{DISCUSSION}

Primary malignant tumor of the liver is categorized into hepatocellular carcinoma (HCC), CCC, sarcoma and lymphoma, and etc. The majority of primary malignant tumor of the liver is $\mathrm{HCC}$ and CCC. The incidence of CCC among primary malignant tumors of the liver is $3 \%$ to $5 \%$, and the incidence of primary hepatic ASC among $\mathrm{CCC}$ is $2 \%$ to $3 \%{ }^{1}$
Pianzola and Drut $^{3}$ described the first case of ASC of the liver in 1971 and computer MEDLINE search revealed about 65 histologically defined primary ASC cases reported in the literature from 1975 to the present. Kobayashi et al. ${ }^{4}$ investigated ASC of the liver from reported cases in the literature, including their case. According to the study, hepatic ASC occurs about two fold more in men and the mean age of hepatic ASC is 62.4 years. Majority of patients complained of abdominal pain as the initial symptom. Abdominal pain, fever, and jaundice appear to be the three most frequent symptoms. Tumors located in the left lobe of the liver in half of the cases.

Few primary hepatic ASC cases were observed in Kormed (http://koreamed.org) search from 2000 to present; the first case presented cholangiocarcinoma with abscess in the liver and was confirmed as ASC postoperatively. Also, the second case was first diagnosed as cholangiocarcinoma and was confirmed as ASC postoperatively. Next case was primary hepatic ASC with formation of tumor-colonic fistula. The forth case was primary hepatic ASC that developed metachronously with a colon adenocarcinoma (Table 1).

The pathogenesis of hepatic ASC remains unknown, with two major hypotheses proposed. Chronic inflammation may cause a metaplastic change in the biliary epithelium that leads to neoplasia. ${ }^{5-7}$ This kind of chronic inflammation of the bile duct is usually associated with infection and/or stones and the lining epithelium of the hepatic cyst. In our case, we can speculate a possible role of persistent inflammation from patient's past history of endoscopic sphincterotomy with stone extraction, due to cholangitis accompanying bile duct stone.

An alternative theory suggests that ASC of the liver arises from the squamous metaplasia of adenocarcinoma. ${ }^{1,2,8-10}$ From this proposal, if metaplatic squamous epithelium subject to malignant transformation, the tumor tissue may be composed of normal epithelium. However,

Table 1. Patient profiles of hepatic adenosquamous carcinoma observed in Kormed (http://koreamed.org) search

\begin{tabular}{ccclll}
\hline Patient no. Age (yrs) & Sex & Clinical presentation & Preoperative biopsy & \multicolumn{1}{c}{ Operation } \\
\hline 1 & 63 & M & Liver abscess & Cholangiocarcinoma & Extended left lobectomy \\
2 & 60 & F & Abdominal pain & Cholangiocarcinoma & Left lobectomy \\
3 & 54 & M & Tumor-colonic fistula & Not done & Extended right lobectomy and right emicolectomy \\
4 & 69 & M & $\begin{array}{c}\text { Metastasis from colon } \\
\text { adenocarcinoma }\end{array}$ & Not done & Hepatic segmentectomy \\
\hline \multicolumn{5}{c}{} \\
\hline
\end{tabular}


most tumor tissue is composed of squamous cell carcinoma and adenocarcinoma cells. Yeh et al. ${ }^{2}$ investigated 12 patients with ASC of the liver and 29 reviewed cases. In their investigation, they speculated a possible role of hepatolithiasis in the malignant transformation superimposed on the squamous metaplasia of CCC. Thus, the second theory seems justifiable for describing the pathogenesis in most cases of ASC of the liver.

In our case, microscopically, the tumor was composed of adenocarcinoma, squamous cell carcinoma, as well as transitional area, including the transformation of adenocarcinoma to squamous cell carcinoma. This is in accordance with the microscopic studies of four other hepatobiliary carcinoma of our institute. The adenocarcinoma component arising from the main biliary duct seemed to differentiate into the squamous cell carcinoma component.

The treatment of choice for this tumor is hepatectomy, and some reports advocated radiation therapy. In our case, the patient was treated with curative operation and postoperative adjuvant chemotherapy. Preoperative diagnosis of hepatic ASC, using fine needle aspiration cytology or biopsy, is crucial for the prognostic prediction. However, due to peritoneal seeding concern and diverse diagnostic sensitivity, preoperative diagnosis of hepatic ASC is difficult. Primary hepatic ASC is initially diagnosed as other hepatic tumors, such as CCC, metastatic carcinoma, HCC, or hepatic cyst. As observed in the possible pathologic mechanism of ASC, our case initially shared features of
CCC clinically. Although hepatic ASC is diagnosed postoperatively, postoperative adjuvant therapy, such as chemotherapy and/or radiotherapy should be started as soon as possible.

\section{REFERENCES}

1. Nakajima T, Kondo Y. A clinicopathologic study of intrahepatic cholangiocarcinoma containing a component of squamous cell carcinoma. Cancer 1990;65:1401-1404.

2. Yeh CN, Jan YY, Chen MF. Adenosquamous carcinoma of the liver: clinicopathologic features in 12 patients and review of the literature. Int Surg 2002;87:125-129.

3. Pianzola LE, Drut R. Mucoepidermoid carcinoma of the liver. Am J Clin Pathol 1971;56:758-761.

4. Kobayashi M, Okabayashi T, Okamoto K, et al. A clinicopathologic study of primary adenosquamous carcinoma of the liver. $\mathrm{J}$ Clin Gastroenterol 2005;39:544-548.

5. Ishak KG, Goodman ZD, Stocker ZD. Tumors of the liver and intrahepatic bile ducts. In: Atlas of tumor pathology, third series fascicle 31. Washington, DC: Armed Forced Institute of Pathology, 2001;259-263.

6. Greenwood N, Orr WM. Primary squamous-cell carcinoma arising in a solitary non-parasitic cyst of the liver. J Pathol 1972;107: $145-148$.

7. Gresham GA, Rue LW 3rd. Squamous cell carcinoma of the liver. Hum Pathol 1985;16:413-416.

8. Arase Y, Endo Y, Hara M, et al. Hepatic squamous cell carcinoma with hypercalcemia in liver cirrhosis. Acta Pathol Jpn 1988; 38:643-650.

9. Moore S, Gold RP, Lebwohl O, et al. Adenosquamous carcinoma of the liver arising in biliary cystadenocarcinoma: clinical, radiologic, and pathologic features with review of the literature. J Clin Gastroenterol 1984;6:267-275.

10. Suzuki E, Hirai R, Ota T, et al. Primary adenosquamous carcinoma of the liver: case report. J Hepatobiliary Pancreat Surg 2002; 9:769-773. 